\title{
Surveillance dosimétrique : résultats des équipages des forces sous-marines de 1989 à 1999
}

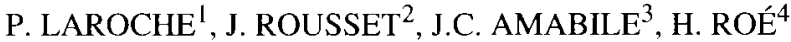

(Manuscrit reçu le 3 mai 2001, accepté le 30 juillet 2001)

RÉSUMÉ Nous présentons le site stratégique de l'Ile Longue et la surveillance médicale et dosimétrique des équipages de sous-marins nucléaires lanceurs d'engins (SNLE) et d'attaque (SNA). Les résultats dosimétriques des sous-mariniers sont colligés sur onze ans. Ils sont comparés à ceux du personnel de la direction des constructions navales et des entreprises extérieures. Sur la période étudiée, ni les marins, ni le personnel civil n'ont été surexposés. Pour tous les groupes, on met en évidence une diminution plus ou moins marquée des doses équivalentes.

ABSTRACT Dosimetric control: report of French submarine forces from 1989 to 1999

We report on the Ile Longue strategic site and describe the medical and dosimetric monitoring of nuclear submarine crews. Over the past eleven years, dosimetric results of nuclear submarine crews have been gathered. We have compared these results to those of workers employed by outside enterprises and the directorate of naval constructions. During this period, neither the crew members nor the workers have been over-exposed. Considering each group, we show a more or less distinct diminution of equivalent doses.

\section{Introduction}

Le sous-marin par sa forme, sa couleur et le milieu dans lequel il évolue, a de tout temps dégagé une aura de puissance et inspiré des sentiments de respect et de mystère. Cette image a été confortée dans le public par de nombreux écrivains dont le célèbre Jules Verne. Les conditions et les doctrines d'utilisation se sont certes modifiées avec les progrès technologiques et l'émergence de l'ère de l'atome, mais il n'existe pas d'arme, de bâtiment de guerre, qui n'ait fait l'objet d'un effort plus constant de la part des nations maritimes. La valeur et l'intérêt de ce type de bâtiment de combat n'ont jamais été remis en cause. Depuis plus de trente ans les sous-marins nucléaires lanceurs d'engins (SNLE) sont la composante principale de la force stratégique française. Ces SNLE, enchaînant à la mer des patrouilles de

\footnotetext{
'Médecin en chef spécialiste du service de santé des armées, Base opérationnelle de l’Ile Longue, 29240 Brest Naval, France.

${ }^{2}$ Médecin des armées, SNLE « L'indomptable », 29240 Brest Naval, France.

${ }^{3}$ Médecin des armées, SNLE « L'inflexible », 29240 Brest Naval. France.

${ }^{4}$ Médecin cn chef, CSS FOST, 29240 Brest Naval, France.
} 
dix semaines avec 111 hommes d'équipage doivent, pour concourir efficacement à la dissuasion, respecter une discrétion absolue. Cette discrétion oblige le médecin à compter essentiellement sur l'équipe médicale réduite qui embarque avec lui, c'est-à-dire un infirmier anesthésiste et un infirmier manipulateur en radiologie. Elle implique pour lui de travailler dans une situation d'isolement total (sans comparaison avec d'autres modes d'exercice), et avec des responsabilités élargies (Laroche et al., 1995).

Outre le suivi médico-chirurgical, la médecine de prévention, la surveillance du confinement et la prise en charge globale des facteurs humains, l'équipe médicale est chargée d'un rôle de radioprotection. Cette dernière mission est primordiale, et elle a fait l'objet d'efforts constants pour amener les doses aux valeurs les plus basses possibles.

La base opérationnelle de l'Ile Longue participe activement à la mission de radioprotection des forces sous-marines. Après avoir décrit brièvement cette base navale complexe et originale, qui sert de soutien global aux SNLE, nous présenterons le principe de surveillance médicale et dosimétrique des équipages embarqués sur ce type de bâtiment de combat. Les résultats dosimétriques des équipages de SNLE des onze dernières années seront présentés et comparés aux résultats dosimétriques :

- du personnel de la DCN,

- du personnel des entreprises extérieures intervenant à bord des SNLE.

\section{L'Ile Longue}

La base opérationnelle de l'Ile Longue est le port base des sous-marins nucléaires lanceurs d'engin. À ce titre :

- elle assure le soutien opérationnel des SNLE en période d'indisponibilité pour entretien (IE) ;

- elle participe à l'organisation et à la mise en œuvre de la radioprotection ;

- elle assure en liaison avec le centre de préparation des équipages des forces sous-marines (CPE/FSM) la formation des équipages dans le domaine de la radioprotection;

- elle coordonne et met en place les moyens en cas d'incident ou d'accident à caractère radiologique ;

- elle organise le suivi radioécologique du site.

Sur ce site cohabitent plusieurs installations nucléaires de base. Elles procèdent aux opérations de maintenance, aux opérations de chargement, déchargement et stockage des éléments combustibles des chaufferies nucléaires embarquées, aux mouvements d'embarquement et de débarquement des missiles stratégiques ainsi qu'à l'assemblage des têtes nucléaires. 


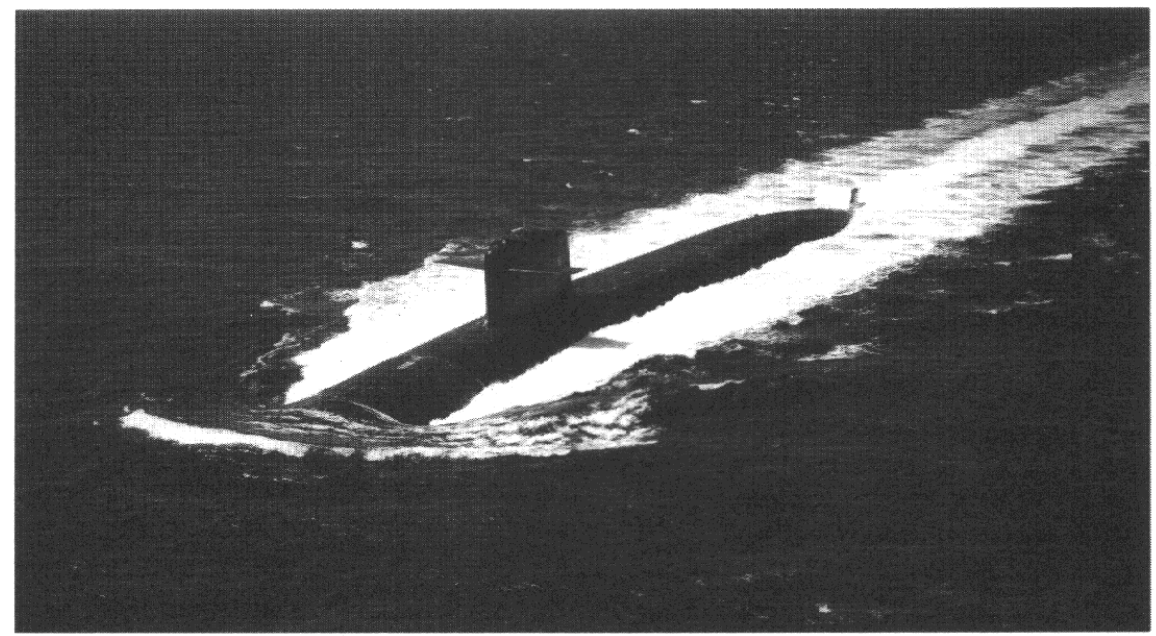

Photo 1 - Le sous-marin nucléaire lanceur d'engins «le Triomphant» en mer. Nuclear submarine "le Triomphant" on the sea.

Afin d'assurer le soutien opérationnel des SNLE engagés dans le cycle, environ 2200 personnes, réparties pour moitié entre civils et militaires, travaillent sur la base. Les activités liées au soutien des SNLE se déroulent vingt-quatre heures sur vingt-quatre afin d'éviter ou de limiter des interactions entre les mouvements ordinaires et les mouvements plus sensibles.

\section{Surveillance dosimétrique à bord d'un SNLE}

\subsection{Les sous-marins nucléaires lanceurs d'engins de nouvelle génération}

Deux sous-marins de type «le Redoutable» sont encore en service. Ils sont remplacés progressivement par des sous-marins de type «le Triomphant», plus rapides, plus silencieux naviguant plus profond (Photo 1). L'équipage, d'une moyenne d'âge de 27 ans, comprend 111 hommes dont 15 officiers. Équipés d'un réacteur nucléaire, ces sous-marins sont armés de torpilles, de missiles à changement de milieu et de 16 missiles stratégiques emportant chacun 6 têtes nucléaires. La radioprotection sur un SNLE concernera non seulement la surveillance du réacteur (protégé par une triple barrière) et des armes stratégiques mais également la surveillance de l'hôpital qui comprend deux appareils de radiologie conventionnels (Photo 2), ainsi que la gestion des différentes sources radioactives d'étalonnages. 


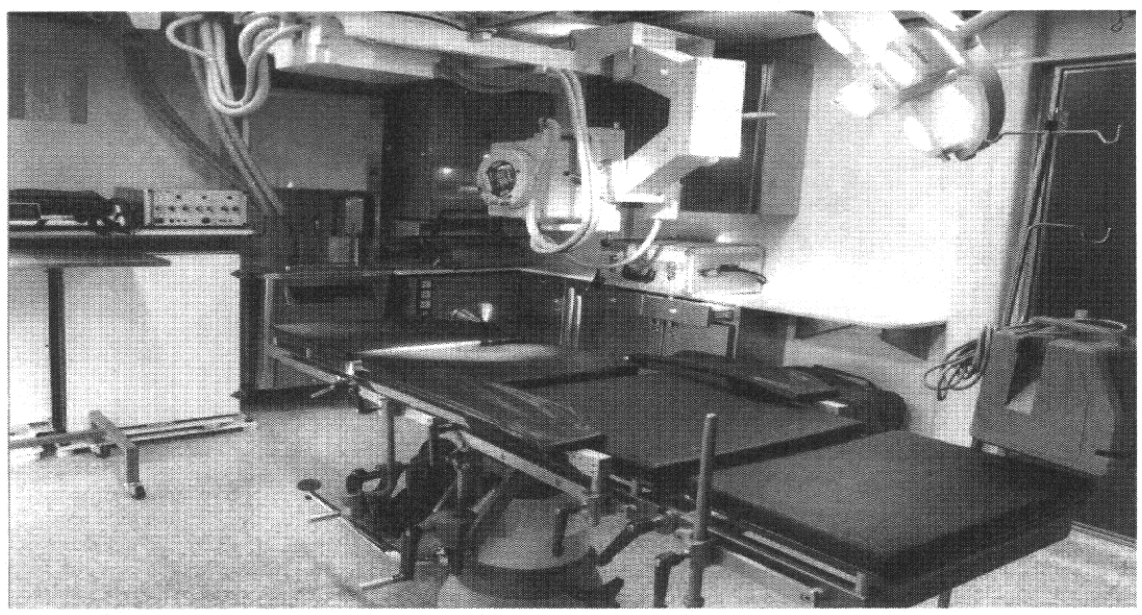

Photo 2 - Le bloc opératoire du SNLE «le Triomphant ».

Surgery of nuclear submarine "le Triomphant".

\subsection{Détecteurs et dosimétrie d'ambiance}

Plusieurs détecteurs fixes surveillent en permanence les expositions potentielles induites par le réacteur ou les missiles stratégiques (Fig. 1). Il existe quatre types de détecteurs (détecteur pour la surveillance des aérosols, détecteur gamma, détecteur bêta gaz, détecteur neutron). En patrouille, les systèmes de surveillance de la contamination atmosphérique n’ont jamais révélé de valeurs anormales.

Ce dispositif de détecteurs est complété par une dosimétrie d'ambiance réalisée à l'aide de 20 dosimètres passifs positionnés à poste fixe, 15 films photographiques gamma et 5 dosimètres passifs comprenant un film photographique gamma et une émulsion nucléaire pour les neutrons (Fig. 2). La disposition des dosimètres d'ambiance, tout en permettant une éventuelle reconstitution post-accidentelle, donne une estimation de «l'ambiance dosimétrique ». Ce dispositif est complété par des systèmes de surveillance de la contamination atmosphérique alpha, bêta et gamma.

\section{Radioprotection des personnes}

\subsection{Réglementation, zonage et entraînement}

\subsubsection{Réglementation}

La protection des personnes contre les rayonnements ionisants au sein de la défense obéit à la réglementation nationale relative à la protection des travailleurs 


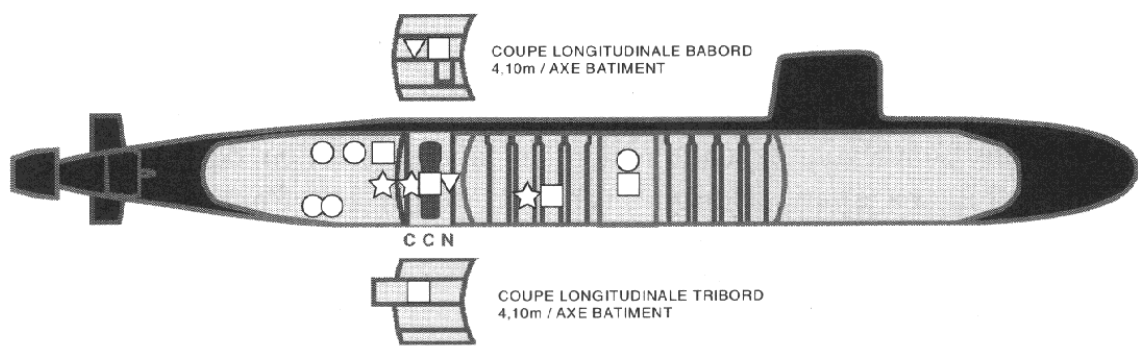

$\nabla \quad$ Surveillance AEROSOLS Détecteur GAMMA

$\bigcirc$ Détecteur BETA GAZ D Détecteur NEUTRON

Figure 1 - Détection à bord des sous-marins type "le Triomphant » (détecteurs fixes). Detection on board submarines class "le Triomphant" (fixed detectors).

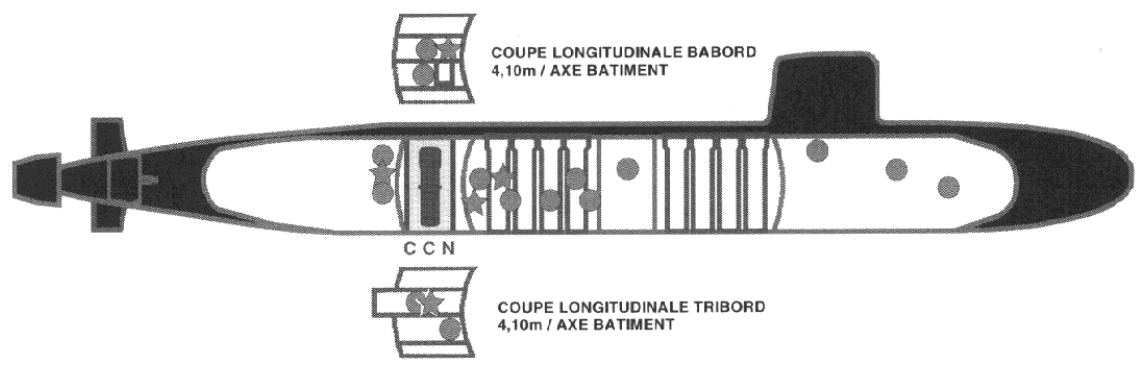

Emplacement des DOSIMETRES

Dosimètre photographique $\gamma$ Dosimètre photographique $\gamma+$ Dosimètre neutrons (émulsion)

Figure 2 - Dosimétrie d'ambiance à bord des sous-marins type «le Triomphant ». Ambience dosimetry on board submarines class "le Triomphant".

contre les rayonnements ionisants, qui s'appuie sur le code du travail et le code de la santé.

Elle est définie par des instructions ministérielles qui fixent les modalités pratiques d'application à la défense de la réglementation de droit commun.

L'ensemble de la réglementation est réuni dans le bulletin officiel édition méthodique (BOEM 627*, 1987) et l'instruction ministérielle 4900 (1993). 


\subsubsection{Zonage et balisage}

En raison de l'espace restreint, le zonage et le balisage mis en place sur ce type de sous-marins sont évolutifs en fonction de la configuration de la chaufferie nucléaire. Le zonage varie selon que la chaufferie est en arrêt froid, en arrêt chaud, en arrêt chaud critique ou marche en puissance $<15 \%$ ou marche en puissance $>15 \%$.

\subsubsection{Entraînement}

Les équipages d'un SNLE bénéficient d'un entraînement de plusieurs semaines (simulateurs à terre), avec remise à niveau des connaissances et évaluation, avant chaque départ en patrouille. La radioprotection (formation et information) est intégrée au programme de cet entraînement.

\subsection{Catégorisation des équipages}

L'ensemble de l'équipage d'un SNLE est de catégorie radiologique A. Ce type de bâtiment vient de bénéficier d'une campagne de mesures gamma et neutrons, afin de permettre une évaluation prédictive de la dose.

\subsection{Surveillance des équipages}

Chaque membre de l'équipage possède, en fonction de son poste de travail et de son service, un numéro spécifique appelé repère. Cette gestion par repère facilite les intercomparaisons des résultats entre SNLE. La dosimétrie individuelle s'effectue à l'aide d'une dosimétrie individuelle photographique poitrine (associée à un film poignet pour certains postes de travail), pour la surveillance de l'exposition externe. Pour certaines activités professionnelles imposant un travail en zone contrôlée (activités liées à la mise en ouvre de la chaufferie nucléaire ou des armes stratégiques), une dosimétrie opérationnelle (système dosiview) vient également compléter la dosimétrie passive. La surveillance de l'exposition externe induite par les neutrons s'effectue à l'aide de dosimètres «bulles » adaptés à l'énergie du rayonnement incident.

Pour ce qui concerne la surveillance de l'exposition interne, l'équipage bénéficie d'une anthroporadiamétrie avant et après une patrouille; un examen radiotoxicologique des urines est réalisé pour un cinquième environ de l'équipage, par échantillonnage, à chaque retour de patrouille. La surveillance médicale est semestrielle. 
TABLEAU I

Dosimétrie photographique individuelle des sous-marins SNLE M4, SNLE NG, SNA de 1989 à 1999.

Individual photographic dosimetry of submarines SNLE M4, SNLE NG, SNA from 1989 to 1999.

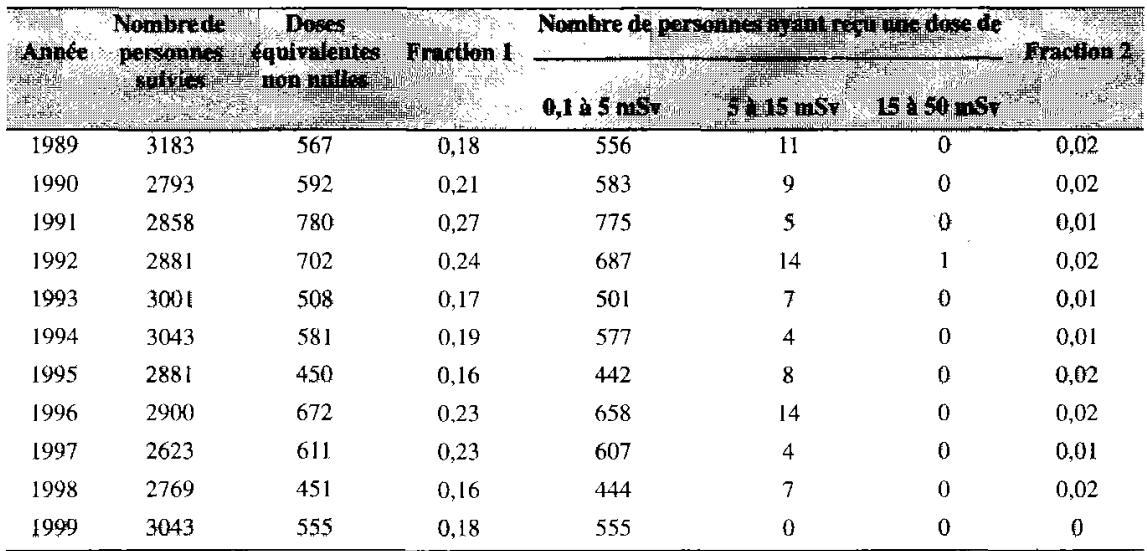

Fraction 1 : nombre de personnes doses équivalentes non nulles/nombre de personnes suivies.

Fraction 2 : nombre de personnes doses de 5 à $50 \mathrm{mS}$ /nombre de personne doses équivalentes non nulles.

\subsubsection{Dosimétrie photographique individuelle}

En plus d'une surveillance des locaux par dosimétrie d'ambiance, des films dosimétriques individuels sont analysés par le service de protection radiologique des armées (SPRA). Les résultats sont fournis dans le respect des règles de confidentialité aux autorités chargées de la surveillance. Les résultats de la dosimétrie sont systématiquement fournis et commentés lors de la visite médicale. Les résultats de la dosimétrie photographique individuelle des sous-mariniers sur onze ans et leur évolution sont présentés au tableau I et sur les figures 3 et 4 . La proportion de doses non nulles décroît sur la période étudiée ainsi que la fraction de doses supérieures à $5 \mathrm{mSv}$. En 1999, aucune dose n'a été supérieure à $5 \mathrm{mSv}$, limite considérée pour le public à cette époque. Pour les sous-mariniers, $80 \%$ des doses équivalentes relevées sont inférieures en 1999 à $1 \mathrm{mSv}$, la nouvelle limite « public » (décret n²001-215).

Les figures 5 et 6 présentent, respectivement, l'équivalent de dose moyen et maximal du personnel travaillant pour la Défense de 1989 à 1999.

Quelques remarques peuvent être effectuées (Laroche et al., 2001) :

- l'équivalent de doses moyen et maximal a significativement baissé depuis 1996 pour les entreprises extérieures et la DCN. Cette baisse peut être attribuée à la mise en place d'une démarche ALARA (As Low As Reasonably 


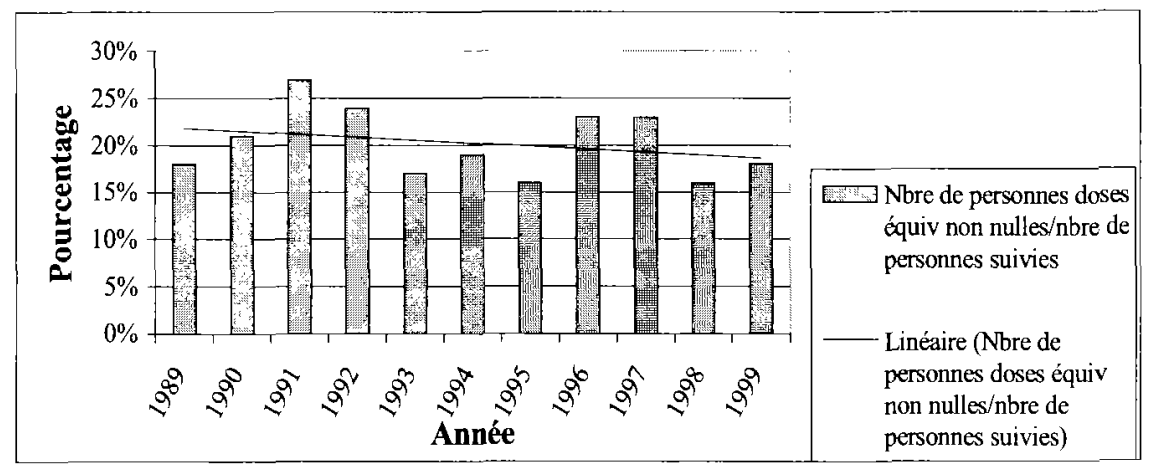

Figure 3 - Proportion de doses équivalentes non nulles : évolution sur onze ans.

Doses superior to $0.1 \mathrm{mSv}$ in proportion to null doses: evolution over eleven years.

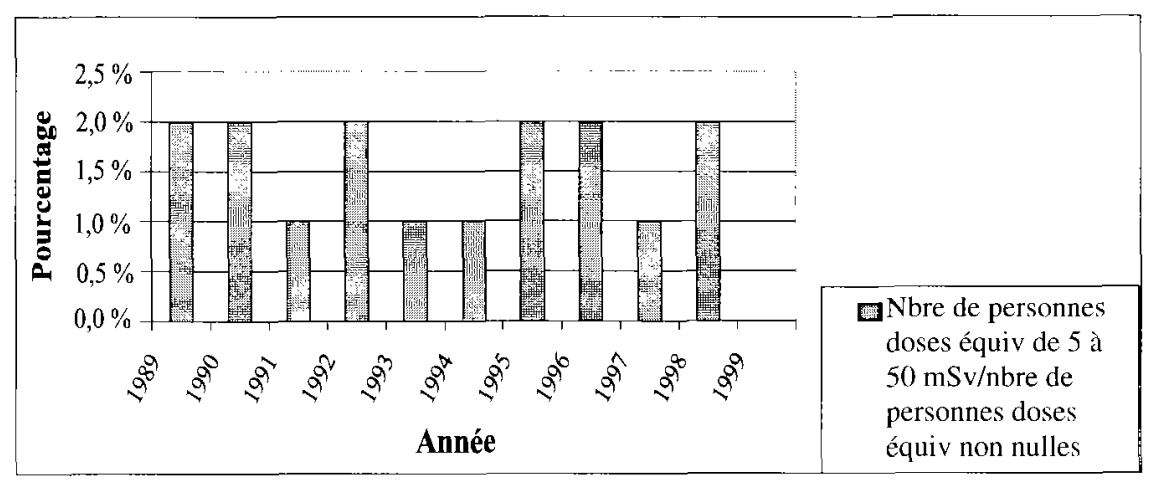

Figure 4 - Proportion de doses équivalentes supérieures à $5 \mathrm{mSv}$ : évolution sur onze ans.

Doses superior to $5 \mathrm{mSv}$ in proportion to doses superior to $0.1 \mathrm{mSv}$ : evolution over eleven years.

Achievable) dans la Défense mais également à la modification du format opérationnel des SNLE (passage de 6 à 4 SNLE) ;

- les personnels de la DCN ont des équivalents de doses moyens ou maximaux plus élevés que les entreprises extérieures ;

- les variations sinusoïdales constatées pour les personnels de la DCN et des entreprises extérieures sont liées aux opérations de démantèlement des premiers SNLE et dépendent du nombre d'indisponibilités pour entretien longues effectuées par an, ces personnels effectuant de la maintenance et des interventions ;

- les courbes pour les personnels des sous-marins sont assez régulières. Ce personnel effectue de la conduite et des réparations; 


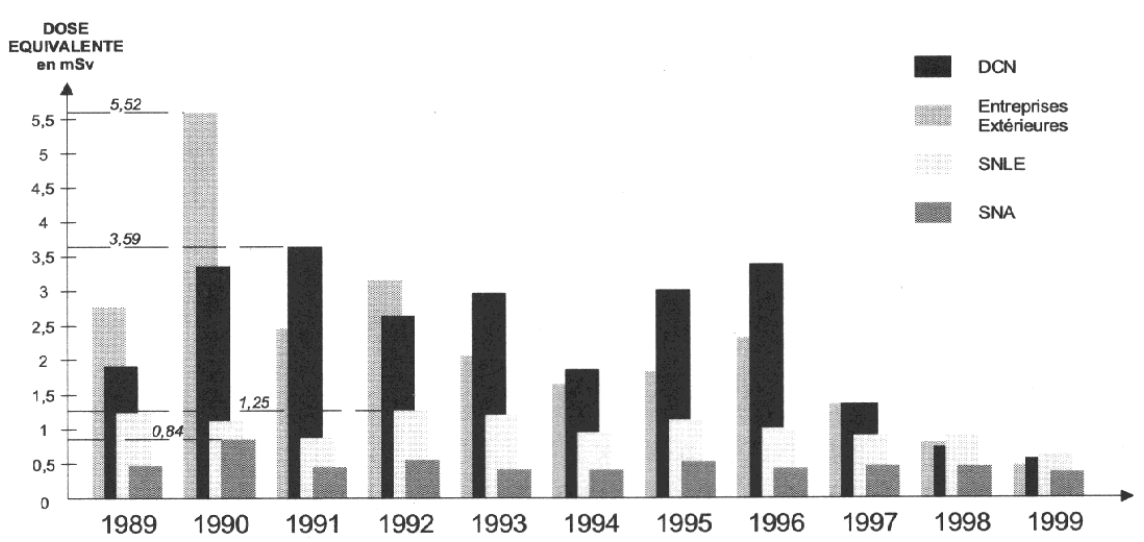

Figure 5-Évolution de l'équivalent de dose moyen du personnel travaillant pour la défense (la dose équivalente moyenne est calculé en ne prenant en compte que les doses équivalentes non nulles). Evolution of the medium equivalent dose of crews and workers (medium dose is calculated with doses superior to $0.1 \mathrm{mSv}$ ).

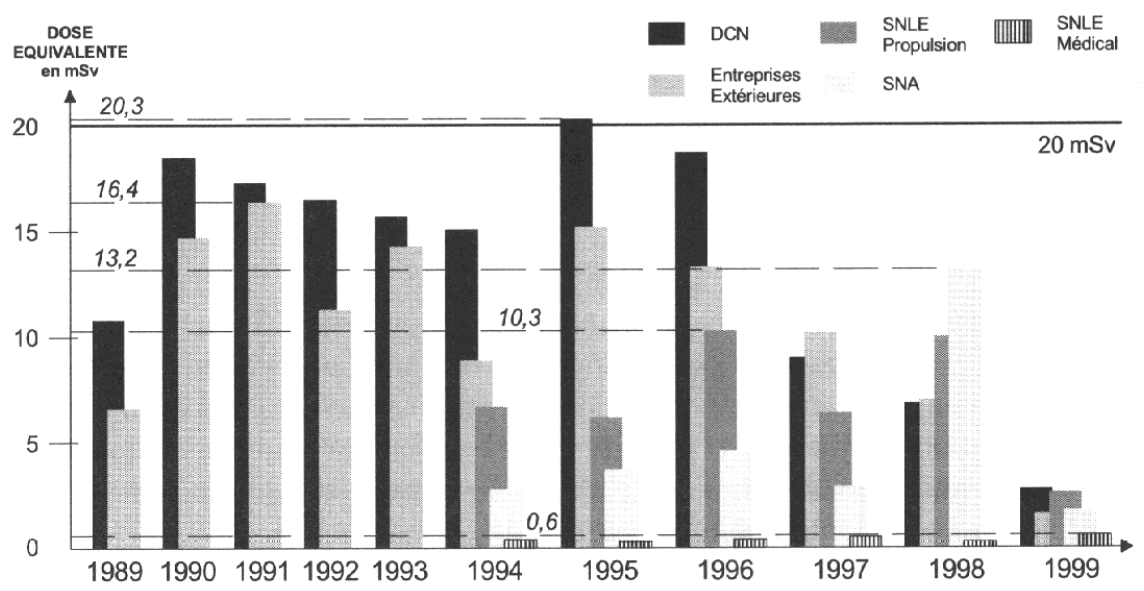

Figure 6 -Évolution de l'équivalent de dose maximal du personnel travaillant pour la défense.

Evolution of the maximal equivalent dose of crews and workers.

- les résultats de l'ensemble du personnel respectent d'ores et déjà les normes imposées par la directive européenne 96/29 (directive EURATOM, 1996), fixant des normes de bases plus restrictives quant à la protection sanitaire de la population et des travailleurs contre les dangers résultant des rayonnements ionisants. Ces nouvelles normes pour les travailleurs sont en cours de transposition en droit français. 
TABLEAU II

Dosimétrie individuelle neutron des SNLE M4 et NG en 1999. Individual neutron dosimetry of submarines M4, NG in 1999.

\begin{tabular}{|c|c|}
\hline 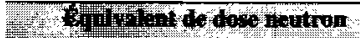 & 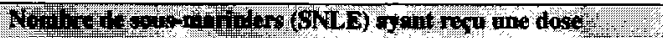 \\
\hline de 0 à $0,1 \mathrm{mSv}$ & 57 \\
\hline de 0,1 à $5 \mathrm{mSv}$ & 173 \\
\hline supérieure à $5 \mathrm{mSv}$ & 0 \\
\hline
\end{tabular}

\subsubsection{La dosimétrie neutrons}

La dosimétrie des neutrons est assurée :

- par le suivi de l'ambiance au moyen de détecteurs neutrons ;

- par une dosimétrie opérationnelle assurée par l'utilisation de dosimètres «à bulles».

Les résultats de la lecture de ces dosimètres sont communiqués au SPRA. Le tableau II présente les résultats individuels neutron des SNLE en 1999.

\subsubsection{Les doses « mixtes » gamma et neutrons des équipages}

La dose cumulée maximale pour l'année 1999 était de 1,4 mSv. Dans tous les cas de cumul, la dose neutron reste inférieure à la dose gamma.

\subsubsection{La surveillance médicale}

Un examen médical a lieu semestriellement pour le personnel de catégorie A et annuellement pour les personnels de catégorie B. Le suivi médical du personnel est basé sur un entretien, un examen clinique et un bilan biologique. La surveillance de l'exposition interne s'effectue à l'aide d'examens radiotoxicologiques (examens des urines des 24 heures complétés d'examens de selles, si nécessaire) et à l'aide d'examens d'anthroporadiamétrie.

\section{Les examens d'anthroporadiamétrie}

32582 examens d'anthroporadiamétrie ont été pratiqués au centre médical de l'escadrille des sous-marins nucléaires lanceurs d'engins (ESNLE) entre 1990 et 2000 : 27 cas de contamination externe (soit moins de $1 \%$ ) au cobalt 60 ont été découverts pour le personnel civil ou militaire entre 1990 et 1999. Parmi ces 27 cas, 13 personnes avaient une contamination interne, mais à un niveau d'activité maximal de $2600 \mathrm{~Bq}$ de ${ }^{60} \mathrm{Co}$, moins de $0,1 \%$ de la limite annuelle d'incorporation (LAI). Ces contaminations sont survenues lors de travaux d'entretiens majeurs nécessitant des interventions sur le circuit primaire. 
Pour l'anecdote, un cas de contamination interne au césium 137 a été relevé chez un militaire avant tout embarquement sur SNLE. Il s'agissait d'une contamination induite par la consommation de grandes quantités de champignons cueillis dans les Vosges. Ces champignons ayant été contaminés par les séquelles des retombées aériennes de l'accident de Tchernobyl.

\section{Les examens de radiotoxicologie}

Une surveillance par des examens radiotoxicologiques des urines est systématiquement pratiquée à chaque retour de patrouille, sur un échantillon de 20 personnes du sous-marin concerné. Ces examens sont destinés à rechercher des signes de contamination interne. La recherche des émetteurs gamma, alpha et bêta (tritium et strontium 90) est réalisée au SPRA. Les émetteurs gamma et alpha sont non significatifs, en revanche les examens pratiqués révèlent toujours la présence d'une activité de tritium urinaire comprise en général entre quelques dizaines et quelques centaines de becquerels par litre (pour mémoire l'excrétion urinaire d'un sujet qui atteint une LAI est d'environ $1,2 \times 10^{6}$ becquerels par litre). De tels niveaux de tritium dans les urines ont également été trouvés chez des habitants d'autres régions n'ayant jamais embarqué sur SNLE.

\section{Conclusion}

La radioprotection est une priorité permanente des forces sous-marines. La présentation de onze ans de suivi dosimétrique et radiotoxicologique permet d'affirmer que sur la période étudiée, aucun accident de surexposition ni de dépassement des limites réglementaires n'a été constaté chez les personnes participant à la mise en cuvre de ces bâtiments de guerre. Les futures normes plus basses imposées par la directive européenne 96/29 sont d'ores et déjà respectées.

\section{RÉFÉRENCES}

Bulletin officiel des armées - édition méthodique, $n^{\circ} 627^{*}$ du $1^{\text {cr }}$ septembre 1987, Sécurité radiologique et protection contre les rayonnements ionisants.

Décret $n^{\circ} 2001-215$ du 8 mars 2001 modifiant le décret $n^{\circ} 66-450$ du 20 juin 1966 relatif aux principes généraux de protection contre les rayonnements ionisants.

Directive 96/29 EURATOM du conseil du 13 mai 1996, fixant les normes de bases relatives à la protection sanitaire de la population el des travailleurs contre les dangers résultant des rayonnements ionisants, J.O.C.E., 29 juin 1996.

Instruction ministériclle $n^{\circ}$ 4900/DEF/CM.2/DR relative à la sécurité nucléaire, édition du 8 février 1993.

Laroche P., Panet J.J., Abiliou R., Lemaire I.. (1995) Médecin de sous-marin nucléaire, Médecine et Armées 23, 667-669.

Laroche P., Rousset J., Defrance J.-P., Grivart de Kerstrat, Amabile J.-C., Féraud M., Ró́ H. (2001) Aspect de la radioprotection dans les forces sous-marines, Médecine et Armées 29 (2), 157-163. 